\title{
Electromyogram (EMG) Driven System Based Virtual Reality for Prosthetic and Rehabilitation Devices
}

\author{
Adel Al-Jumaily \\ School of Electrical, Mechanical and Mechatronic \\ Systems, Faculty of Engineering, University of \\ Technology, Sydney, Australia \\ PO Box 123 Broadway NSW 2007 Australia \\ adel@eng.uts.edu.au
}

\author{
Ricardo a. Olivares \\ School of Electrical, Mechanical and Mechatronic \\ Systems, Faculty of Engineering, University of \\ Technology, Sydney, Australia \\ PO Box 123 Broadway NSW 2007 Australia \\ ricardo.a.olivares@student1.uts.edu.au
}

\begin{abstract}
The users of current prosthetic and rehabilitation devices are facing problems to adapt to their new hosts or not receiving any bio-feedback despite rehabilitation process and retraining, particularly when working with Electromyogram (EMG) signals. In characterizing virtual human limbs, as a potential prosthetic device in 3D virtual reality, patients are able to familiarize themselves with their new appendage and its capabilities in a virtual training environment or can see their movements' intention. This paper presents a Virtual Reality (VR) based design and implementation of a below-shoulder 3D human arm capable of 10-class EMG based motions driven system of biomedical EMG signal.

The method considers a signal classification output as potential control stimulus to drive the virtual prosthetic prototype. A hierarchical design methodology is adopted based on anatomical structure, congruent with Virtual Reality Modeling Language (VRML) architecture. The resulting simulation is based on a portable, self-contained VR model implementation paired with an instrumental virtual control-select board capable of actuating any combinations of singular or paired kinematic 10-class EMG motions. The built model allows for multiple degree of freedom profiles as the classes can be activated independently or in conjunction with others allowing enhanced arm movement.
\end{abstract}

\section{Keywords}

Virtual Reality, EMG signal, prosthetic and rehabilitation.

\section{INTRODUCTION}

The human nervous system is a complex neurological scheme capable of processing almost an infinitesimal number of expedient neurological signals within short temporal periods. The peripheral sensory nervous system is able to communicate sensory and motor information with muscles, skin tissue, and organs to the central control system (CNS). The localized peripheral nervous system can be harnessed for actuation of a non-existent limb through virtual limb via a direct EMG control (also known as myoelectric control) signal for amputees or those with congenital defects. The surface electromyogram (SEMG) used to collect all motor unit action potentials (MUAP) within the immediate skin area of SEMG electrodes. For electromyographic (EMG) prosthesis actuation to be effective, it must be highly responsive, reliable and predictable when associated with predetermined motion sets [1]. A framework model parts for such system, usually, is specified by EMG acquisition, processing, feature extraction, and classification Electromyographic signals can be processed for input control of smart biomechanical prosthetic devices, ideally in the temporal vicinity of real-time response, as a utile replacement of lost or severed limbs $[1,2,3,4,5,6]$. Advances in Electromyography and signal processing have led to a host of researchers and technologists developing "smart" EMG controlled prosthetic devices capable of mimicking actual limbs by sensing and processing surrounding peripheral nervous system EMG signals. The challenge of these biomechanical devices is to operate within human tolerances for time delays and mobility, so the host does not reject the new appendage, rather functionally replaces the actual one. In this paper VR model is build based on a virtual characterization of the human arm into a control system comprised of complementary actuating muscles groups, peripheral neurological sub-networks, and EMG signals and bone-tissue physical framework. This model is emulate the virtual prosthesis graphical user interface, provide visualization by means of a multiple degree-of-freedom (DOF), and has a graphical user interface 3D re-creation and simulation of the below shoulder human arm as a prototype which is characterized to move within ten predetermined classified electromyogram motion profiles ,recorded by 16 SEMG surface electrodes attached to the perimeter of the upper arm that used, to create stimulus for a virtual reality prosthesis implementation synthesized in VR. EMG processing and classification had been done in our previous work in [2].

This paper organized, in section two background of EMG and VR and related work are highlighted, propose model specifications and details taking in account the limitation of the hand physical movement is explained in section 3 , while section four details the methodology and implementation of the model. The paper concluded in section 5 .

\section{BACKGROUND AND RELATED WORK}

Many of our senses are stimulated by Virtual Reality (VR) as it describes a reality emulated by computer generation of virtual objects to create a three-dimensional virtual environment $[7,8]$. Inputs to the users' sensory organs are deceived in such a manner as to convince the subject they are actually in the Virtual Environment rather than the physical one. The user is immersed in a virtual world which he/she can manipulate using hardware interfaces to a software self-contained system. The actual living world is three dimensional therefore it is natural for perception actualization to be rendered in a three dimensional system. Cognition processes in the brain are circumspect in a three dimensional perspective, hence a three dimensional virtual reality would be better suited to our natural sensory mechanisms and would involve less processing for conceptual adaptation [8].

The work in this paper can be sought ideally around the integration of Virtual Reality modeling/simulation and surface sensing (SEMG) acquired Electromyogram motion signals of the 
human arm. The EMG signal is a small electric current that is generated when a muscle is contracted [9]. The unit potentials of EMG are directly related to the embodiment of the motor unit and its inherent number of muscle fibers per motor unit, together with their metabolic muscle types [10]. The research and knowledge base in early stages for this area was established by developed a 3D virtual model of the knee driven by EMG Signals in skeletal to determine muscle controls via the neuromuscular system[5]. While other work was aim to validate and confirm qualitatively the knee's dynamic mechanical properties and forces necessary for practical operation [6]. The work in [6] had made a historical claim for beginnings and futuristic advances in cybernetics by laying the foundation for the need of advances in intelligent artificial limbs. The work in [1] explains the complex kinematic structure of man, making comparisons to potential Humanoid Robots of great complexity and large degrees of freedom (DOF) necessary to mimic human movement.

Virtual Reality prosthetic limb recreations designed by biomedical researchers are often used for assisting amputee or congenital defected patients, and are modeled in a kinematic virtual space mimicking their natural counterparts within tolerances of DOF and specified mobility [1,3]. Some of the works done in this area was a virtual modeling project that took a forwards dynamic approach by considering knee muscle groups and their interactive forces in trajectory motion to develop a sustainable mechanical model. The simulation in virtual reality was only a step in the process of ultimately implementing a real-time EMG-driven exoskeleton knee geared for rehabilitation purposes. The work was limited the EMG acquisition to two signals for control of a virtual knee to keep the model within practical workable bounds and for simplicity. Emphasis was greatly placed on mechanical and kinematic system boundaries, limitations and force controls used to control the realistically biomechanical virtual implement [6]. Their testing phase was conducted in reference to knee torsion control using a two stage process of unitary flexor muscle contraction and dual flexor and extensor muscle contraction in order to verify the simulation models' torsion trajectories.

The work in [4] outlines the development of a virtual reality environment for designing and fitting a neural prosthetic limb. In this model a subject could manipulate the virtual limb to operate with virtual objects so as to gain knowledge and custom on its operability in an effort to enlighten the limbless patient with his/her new prosthetic attachment. The work able to comprehensively ascertain exacting hardware provisions (such as for neuro-hardware interactivity) and provide clear guidelines for potential future designs.

VR concept creations, in all manifestations, stood to re-create human form and its biomechanical composure more or less to an accurate degree without compromising form, agility, human limitations and biomechanical degrees of freedom (DOF). Inspired researchers took to better the existing human form by envisaging cyber-like EMG stimulated bionic bodily components which could far surpass the rudimentary and often monotonous parts of the existing human body such as the expansive VRE modeling by [4]. Many software packages currently exist in the marketplace for the creation of a virtual reality 3D animation, [8]. VRML modeling language, which is a component of MATLAB v7 virtual reality toolbox add-on can be used to both create, manipulate, animate and view 3D models, the software also allows for direct integration to Simulink for control system signals. Fine manipulations can be achieved using the form of 3D modeling tool uses intricate coordinate timing interpolations to recreate real-life behavior of autonomous systems.

\section{PROPOSE MODEL SPECIFICATIONS}

The work implementation of this paper is based on simplified human arm kinematics and executes 10-class EMG based motions [2]. Hence, the objective ten motions chosen for modeling were: Forearm Pronation, Forearm Supination, Wrist Extension, Wrist Flexion, Wrist Radial Deviation, Wrist Ulnar Deviation, Ball Grip, Release, Hand Open, and Hand Rest State that are shown in Figure 1.

\subsection{Kinematics}

Kinematics, in VR, is a description of body motion with consideration to time, velocity, acceleration and displacement. Thus, it is an area that describes purely the temporal and spatial components of motion without taking into consideration the conditions warranting the motion. In a sense, it can be described as pure motion devoid of energy, momentum or force or any other causes, as modeled in this work.

Kinematics represents the $3 \mathrm{D}$ object parameters operated to control its transformations. These parameters include translation position, rotation angle and scaling magnitude of an object in the $X, Y, Z$ coordinate space.

Rotational kinematics has particular significance in this paper as the 10-class motion profiles are implemented through rotational interpolation of object nodes along finite kinematic rotational angles. Thus, it is possible to describe the required 10-class EMG motion profiles with orientation descriptions about an axis using angular position and angular velocity quantities as Figure 2 shown. The angular position of any motion is the oriented displacement from a selected origin on the axis of rotation to an object point named vector $\mathrm{r}(\mathrm{t})$ as illustrated in Figure 2 . Perpendicular to the rotational axis is vector $\boldsymbol{r} \perp(t)$ which is component of $\mathrm{r}(\mathrm{t})$. The right-hand rule specifies the angular position of a point as the angle $\theta_{k}$ (radians) from the reference axis to the vector $\boldsymbol{r} \perp(t)$ in a counter clockwise motion. Therefore, the point angular position of an EMG class motion can be described as $\theta_{k}$ from the rest position. The distance $S$ travelled by the object in rotational motion away from the origin can be described as

$$
s=\theta_{k} \boldsymbol{r}(t)
$$

Akin to linear motion, the angular velocity is measured in respect to angular distance over time,

$$
\omega=\frac{\Delta \theta_{k}}{\Delta t_{i}} \quad\left(\mathrm{rads}^{-1}\right)
$$

where: $\Delta \theta_{k}$ represents the angular position relative to axis $k, \Delta t_{i}$ represents motion time interval, and $\omega$ represents angular velocity. To satisfy the 10 -class motion profiles, the rotational points of origin for each motion will be about the limb spherical joint and the class motion rotations are axially singular dependent and within the human arm's natural anatomical limitations.

\subsection{Anatomy of the Human arm}

The human arm has 7 DOF thus the shoulder permits pitch, yaw and roll. The elbow permits pitch, and a wrist allows for pitch, yaw and roll. The flexor and extensor muscle groups work in tandem in the upper arm as complementing opposite pairs in order 

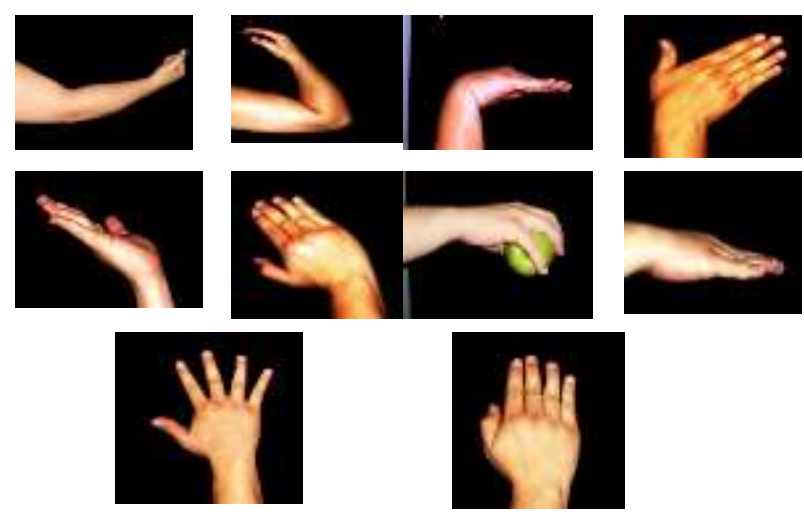

Figure 1 - Forearm movements.

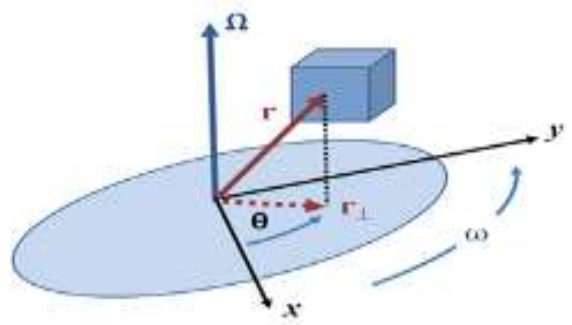

Figure 2- Rotational kinematic orientation

to rotate the elbow joint. Flexion in the human arm is achieved by contracting the biceps brachii whilst extension is achieved by the contraction of triceps brachii. The triceps brachii are posterior to the arm skeleton and is appended behind the pivot point permitting elbow flexion. In opposing fashion, elbow flexion is achieved by the anterior biceps brachii which is appended to the front of the pivot point.

The anatomy of the wrist is considerably more complex than the other joints in the body, as it is composed of eight immediate carpal bones arranged in two rows. The wrist has the ability of a complete range of motion as well as being robust for lifting and gripping strength. Tendons, which are nascent in the forearm, control wrist motions and are connected to the surrounding bone structure. Flexor tendons cross the anterior part of the wrist and create wrist bending and curling of the digits. The opposite motion is achieved by Extensor tendons which are located within wrist compartments. In the VR model, the elbow joint is replaced by a one DOF rotation representing the class motions of Elbow Pronation and Supination; while the Wrist Joint is modeled on a two DOF rotational node capable of four EMG class motions; namely Wrist Flexion, Wrist Extension, Wrist Ulnar Deviation and Wrist Radial Deviation.

The anatomical human hand is made up of twenty-four muscle groups which provide for an extensive combination of motions. Out of the three hand movements (fixation, slow-to-rapid, ballistic); fixation movements, where the hand is held in a static kinematic position by promoting and opposing muscles groups, are considered in the scope of this work. Mechanically, six DOF are afforded by the hand in linear $X_{v} Y_{v} Z$ motions in addition to

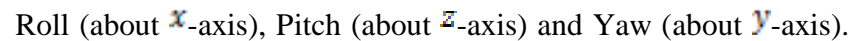
The anatomy of the human elbow, wrist, and hand are shown in Figure 3Error! Reference source not found. The VR modeling of the right-hand is based on a simplified human hand structure containing all of the digits and

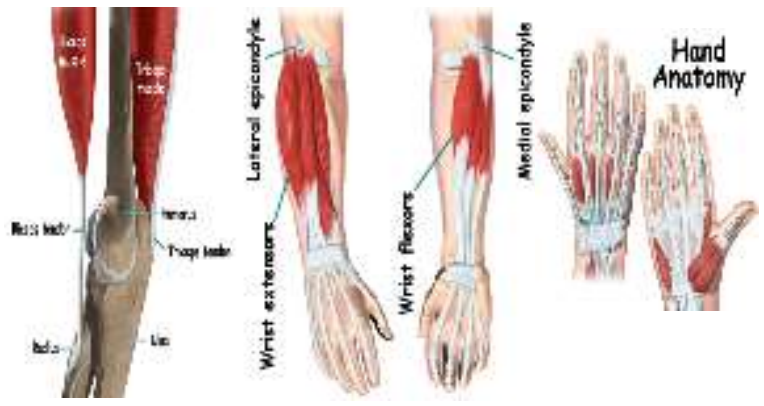

Figure 3-Anatomy of the Elbow, Wrist, and Hand

nodes of an actual hand but limited to the EMG class motions specified. The VR model has the capability to perform Ball Grip, Ball Release, Hand Open, and Rest State motions.

\section{IMPLEMENTATION AND TESTING}

An acquired EMG signal, after processing, is used as a control input to a virtual device. The objective here is to develop a dynamic 3D Virtual Reality Simulation of a human arm capable of ten classes of motion. The dataset and the classification outcome used was obtained through our work in [2]. A methodology is presented in sequential stages which were used to produce the resultant VRML human arm model and its interface.

The recommended dataset contained three DOF wrist motions, two hand grips and a rest state; however in the model planning stages the two grips were combined to form a single Ball Grip and Ball Release. The 3D model is designed to execute each one of these motions individually or in simultaneous combinations of each other. The 3D simulation mimics the neuromuscular actuation of the respective motions' motor unit and emulated by using interactive TouchSensor's embedded into the model. An anatomically logical top-down construction methodology was adopted in synthesizing the VR Arm model. Progressively, the building starts from upper parent hierarchical components down to the lowest children components of the model to ensure realistic continuity and model congruency as the structural representation to create 3D model shown in Figure Error! Reference source not found. 4 .

\subsection{D arm construction}

The simulated RH Arm design embodies a direct correlation to the VRML architectural hierarchy. In order to construct the virtual human arm model, a piece-wise methodical approach was taken based on VRML hierarchical structures.

\subsubsection{Building model stages}

The first stage was comprised of the Upper Arm and Fore Arm indexed face 3D node and the Elbow Joint. The Upper Arm object was located at the coordinate origin $(0,0,0)$ affixed at its lower extremity ( $y$-coordinate) to the Elbow joint solid sphere radius based on anatomically of the Elbow joint, the Fore Arm indexed face object was appended.

\subsubsection{Rotational Joints}

The rotational joints (spheres) were utilized to mobilize adjacent limbs through predetermined angles of rotation. The Elbow joint node was constructed using one degree of rotation about the $Z$ axis (perpendicular to the reference $x y$-plane of the arm construction) in order to achieve Forearm Pronation and Supination (from 0 to 1.129 radians). The Wrist Joint (radius = 
0.024) was created as a lower appendage to the Forearm construction and has two degrees of rotation for Ulnar and Radial Deviation (about $y$-axis) as well as Wrist Extension and Flexion (about $z$-axis).

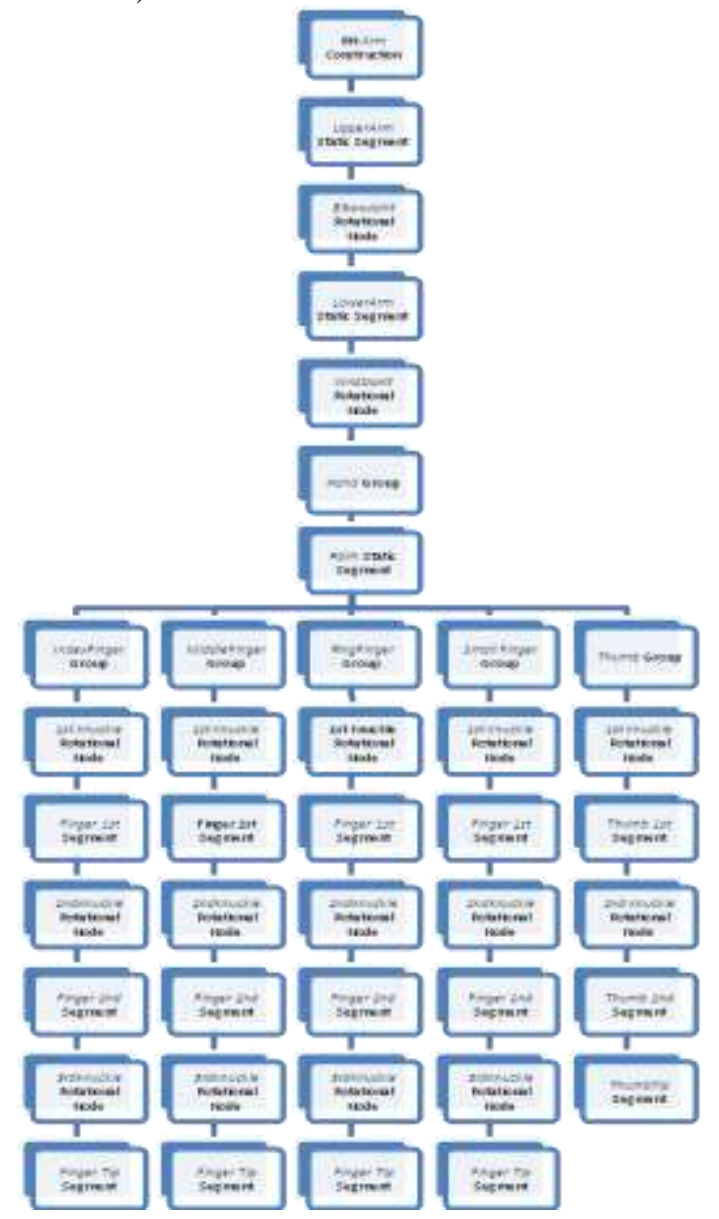

Figure 4 - Hierarchical architecture for VR Arm 3D design

\subsubsection{Hand structure}

The right-hand structure is encompassed as a child grouping transform of the parent transform group Wrist Joint. It is composed of a palm block, thumb and four digits which have intermediary rotating (knuckle) joints for multi-DOF mobility. The five hand digits are composed of cylindrical segments of relative dimensions and intermediary rotating knuckle joints, which provide rotational dexterity to the fingers. The third stage $\theta_{k}=64^{\circ}$ degrees ( 1.129 radius) animation trajectory about the $z$ axis centred on the elbow joint from the default Forearm Supinated relaxed position towards the Upper Arm creating Forearm Pronation. This was achieved by interpolating a sequence of timing fractions, spanning from 0 to $5 \mathrm{sec}$, routed against angular rotation locus.

Additional animation features were added in the fourth stage which included a reversal motion to the preceding model from Forearm Pronation to Forearm Supination, the inclusion of a specific 3D coordinate space point view such as the preliminary Hand View viewpoint for clarity of hand movement observation. The Hand Open and Rest States were achieved by rotating the $\theta_{k}=-15^{\circ}$ about the $x$-axis. The thumb joint was duly rotated $\theta_{k}=-30^{\circ}$ degrees about the $y$-axis to create a ( 3 second) open hand interpolation trajectory. The fifth installment of the building the model revealed the need for the return of hand to Rest State counter-motion animation. In attempting this, some basic adjustments were made to the alignment of the wrist in natural anatomical axial continuance with the lower extremity of the Forearm. Both the Ulnar and $\theta_{k}=+/-45^{\circ}$ respectively from the natural axis of the forearm Radial Deviations were kept to a about the $y$-axis in the $x z$-plane. A more complex motion was accomplished in the building of Ball Grab motion profiles. This was achieved by a sequence of time interpolated motions in which the fingers were spread as Hand Open followed by a rotation of each finger knuckle joint simultaneously about the ${ }^{\mathbb{Z}}$-axis in order to create a clutching effect. The palm knuckle joints, other than $\theta_{k}=+25^{\circ}$ degrees (about $z$-axis). Subsequently the centre $\theta_{k}=-15^{\circ}$ degree rotation of the third knuckle joints to create a natural grip motion. Simultaneously, the thumb-palm knuckle was rotated about a translated axis evenly located mid-between $+y$ $\theta_{k}=+70^{\circ}$ degree rotation of the thumb middle knuckle joint about the $x$-axis to create a natural clutching thumb, as demonstrated in Figure 5

As the specified EMG 10-class motion suite was being populated, a requirement for interim triggers arose in respect to individual EMG motions as particular arm/hand motions combined and clear distinctions assessed for differentiation, and allowing for testing to be conducted. The testing of individual motions' fluidity against other motions was assessed by visually comparing unique motions by generating ten interim color-coded triggers from touch-sensor spheres strategically placed around the 3D virtual model. Each spherical trigger touch-sensor was allotted a unique motion trajectory whilst an adjacent complementing trigger was assigned the reverse pair motion. Also the combinations of simultaneously triggered motions such as Elbow Pronation and Wrist Flexion were successfully tested from several viewpoints for provisional assurance of agility and performance in motion, as shown in Error! Reference source not found.Figure 6.

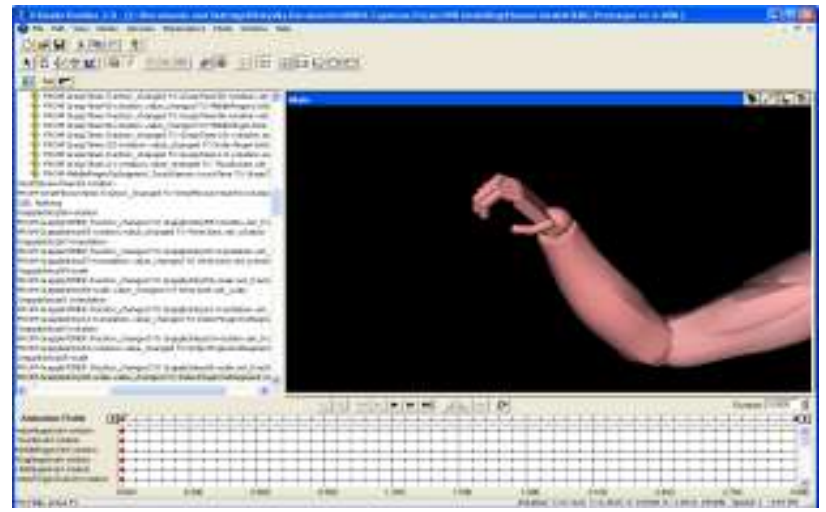

Figure 5 - VRML model of Ball Grip motion animation.

\subsubsection{Motion Timings}

Timing of motions was based around natural arm movement for clear visibility and perception of trajectory. Table 1 represent of orientation interpolated animation timings used in the model for each of the 5 complementing pairs of EMG class motions. In this final stage, the model version has included a place holding billboard control panel that created to always face the user regardless of which perspective viewpoint is chosen. Furthermore, 
three new perspectives were created allowing the user full visibility and control of each motion.

Table 1 - Class Motion animation timings

\begin{tabular}{|c|c|}
\hline Class Motion & Animation Timing \\
\hline Forearm Pronation/Supination & $5 \mathrm{sec}$ \\
\hline Wrist Flexion/Extension & $4 \mathrm{sec}$ \\
\hline Hand Ball Grab/Release & $3 \mathrm{sec}$ \\
\hline Hand Open/Rest & $3 \mathrm{sec}$ \\
\hline Wrist Radial/Ulnar Deviation & $3 \mathrm{sec}$ \\
\hline
\end{tabular}

Final testing and analysis was also conducted at this stage to ensure motions, and all their combinations executed according to specifications with respective control panel prompts. Furthermore, the raw VRML code was examined for stability, repeatability, consistency and balance in architecture. Specified objectives, limitations and exclusions were confirmed and user testing was conducted to meet the required quality, user-friendliness and robustness of the model. Figure Error! Reference source not found.15 illustrates the final model including the selection control board and title.

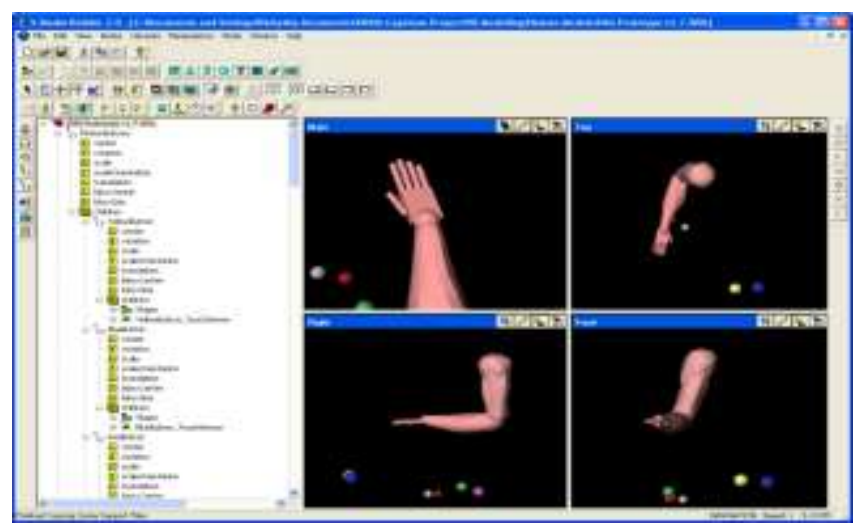

Figure 6 VR model for the multiple perspectives and interim

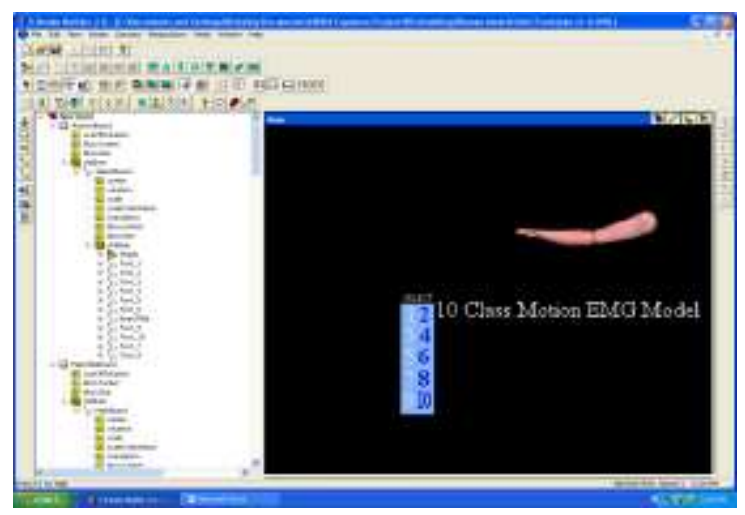

Figure 7 - EMG VR final model

\section{CONCLUSIONS}

The present work has demonstrated successfully built model for the 10-class EMG motions visualization for EMG driven prosthetic or rehabilitation devices. This work was based on research of EMG acquisition, processing, and classifications techniques in order to provide discriminatory signal control input to the virtual prosthetic model. The developed model is consistent with human arm anatomical construction such that basic kinematic theory was used to model the 10-class EMG motions.
The model viewpoints created were strategically placed to capture either pair or motion state of actuation. The work made possible combinations of EMG motions within predefined states. It is ready for receiving the classification signals that can be directly used to power rotational nodes providing the model with multiple DOF.

For future model expandability, clarity and upgrading, each rotational node is uniquely named and characterized independently in group transforms as provision for direct hardware digital control algorithm interfacing. Further complexity could be incorporated into the model by creating actual Indexed Face Sets of the hand and fingers, sound sensors and audio feedback, scenic backgrounds etc. However, over complexity of the environment model would compromise processing performance and possibly hinder GUI responsiveness which would undermine the fundamental objectives of creating a realtime virtual prosthesis. The built model is portable and can be used through TCP/IP External Authoring Interfaces (EAI) over internet connections enabling remote tele-operability or direct remote connections

\section{REFERENCES}

[1] Folgheraiter, M and G Gini. 2003. A Bio-inspired control system and a VRML simulator for an autonomous humanoid arm. IEEE Humanoids 2003. Karlsruhe, Germany.

[2] Al-Jumaily, A and R.N Khushaba. 2007. Fuzzy Wavelet Packet based Feature Extraction Method for Multifunction Myoelectric Control. International Journal of Biomedical Sciences. 2(3), pp.186-194.

[3] SU, Y., A. Wolczowski, M.H. Fisher 2005. Towards an EMG Controlled Prosthetic Hand Using a 3D

Electromagnetic Positioning System. Instrumentation and Measurement Technology Conference. IMTC 2005. Ottawa, Ontario: pp. $261-266$

[4] Hauschild, M, R Davoodi, and G.E Loeb. 2007. A virtual reality environment for designing and fitting neural prosthetic limbs. IEEE Transactions on Neural Systems and Rehabilitation Engineering. 15(1), pp.1534-4320.

[5] Sartori, M, G Chemello, and Pagello.E. 2007. A 3D Virtual Model of the Knee Driven by EMG Signals. LNAI., pp.591601.

[6] Soares, A, A Andrade, E Lamounier, and R Carrijo. 2003. The development of a Virtual Myoelectric Prosthesis Controlled by an EMG Pattern Recognition System Based on Neural Networks. Journal of Intelligent Information Systems.21 (2), pp.127-141.

[7] Medeiros, D.J, J.S Watson, M.S Carson, and M.S Manivannan. 1998. Simulation and Modeling with Artificial Reality Technology (SMART): An integration of VR and Simulation Modeling. 30th conference on Winter simulation.

[8] Tsepkovskiy, Y, L Antonov, CV Kocev, and N Shoylev F Palis. 2008. Development Of A 3d and VRML Virtual Hand Models. Journal of the University of Chemical Technology and Metallurgy. 43(1), pp.159-164.

[9] Saksit. 2008. EMG Thesis. [Online]. [Accessed 05 September 2008]. Available form hyperlink "http://medusa.sdsu.edu/Robotics/Neuromuscular/Theses/Sa ksit/Chapter1.pdf" 
[10] Orsi, C. and H. De Bruin. 2000. Measurement of electrical and contractile properties of motor units using EMG and accelerometry. Proceedings of the 22nd Annual International Conference of the IEEE. Chicago, pp.3015 3017. 